\title{
Mid-Infrared Observations of the Galactic Center Arc and the Sgr A East HII Region
}

\author{
F. Yusef-Zadeh ${ }^{1,2}$ \\ LASP, Goddard Space Flight Center and Northwestern University \\ C.M. Telesco ${ }^{2}$ and R. Decher ${ }^{2}$ \\ Space Science Laboratory, NASA/Marshall Space Flight Center
}

\begin{abstract}
We have used the 20-pixel IR camera to observe thermal IR emission from dust associated with the radio continuum Arc near the Galactic center and the cluster of HII regions in the immediate vicinity of Sgr A East. We detected strong $10 \mu \mathrm{m}$ emission from the eastern and western arched filaments $(\mathrm{G} 0.1+0.08)$, from an unusual pistol-shaped structure known as G0.15-0.05 and from the brightest member of the Sgr A East HII region. Spatial maps of these features at $10 \mu \mathrm{m}$ with a resolution of $4.1 " \times 4.2$ " are presented and are compared with $5-\mathrm{GHz}$ radio images. We find a general spatial correlation between the ionized gas and the dust distributions. The ratio of IR to radio flux densities is significantly different in the eastern and western arched filaments, which suggests that the source of heating has a softer spectrum along the eastern arched filaments. In addition, the ratio of IR to radio flux densities, which is typically $\sim 10$ in normal Galactic HII regions excited by $\mathrm{O}$ stars, is at least a factor of two higher than this value in almost all the sources we have observed. This suggests that additional mechanisms other than trapped Lyman $\alpha$ radiation should be present in heating the dust, e.g. stochastic heating of small dust grains by energetic particles associated with the nonthermal filaments.
\end{abstract}

\section{Introduction}

The radio continuum Arc located near $1=0.2^{\circ}$ consists in part of two prominent ionized features, G0.18-0.04 and G0.1+0.08, which appear to intersect the long nonthermal filaments (Yusef-Zadeh, Morris and Chance 1984). Motivated by the unusual morphology of these thermal features and the possible importance of an interaction between thermal and nonthermal gas, we have searched for $10 \mu \mathrm{m}$ emission from four Galactic center radio sources in order to investigate the spatial relationship between the dust and gas on a scale of a few arcseconds and to gain an insight into the nature of the sources that heat the dust and gas. Two of the observed sources are associated with the radio arched filaments, one is associated with the intersection of the nonthermal filaments with the Galactic plane and one is the brightest component of a cluster of HII regions lying at the eastern edge of the Sgr A East nonthermal source.

In this contribution, we present preliminary results of observations made using the 20-pixel bolometer infrared array camera developed at NASA/Marshall Space Flight Center. The size (FWHM) of each square pixel of the array was 4.1 " $\times 4.2$ ".

1,2 NRC Resident Research Associate

2 Visiting Astronomer at the NASA Infrared Telescope Facility 
Figure 1: Solid and broken lines presented in Figures 1,2,3 and 4 correspond to $10.8 \mu \mathrm{m}$ and $6 \mathrm{~cm}$ emission, respectively. Contour intervals associated with the eastern arched filaments $(\mathrm{G} 0.1+0.08)$ at $10 \mu \mathrm{m}$ and $6 \mathrm{~cm}$ wavelengths are set at 150,200,300, $400,500,600,700 \mathrm{mJy} /$ beam area and 10, 15, 20, 25, $30 \mathrm{mJy} /$ beam area, respectively.

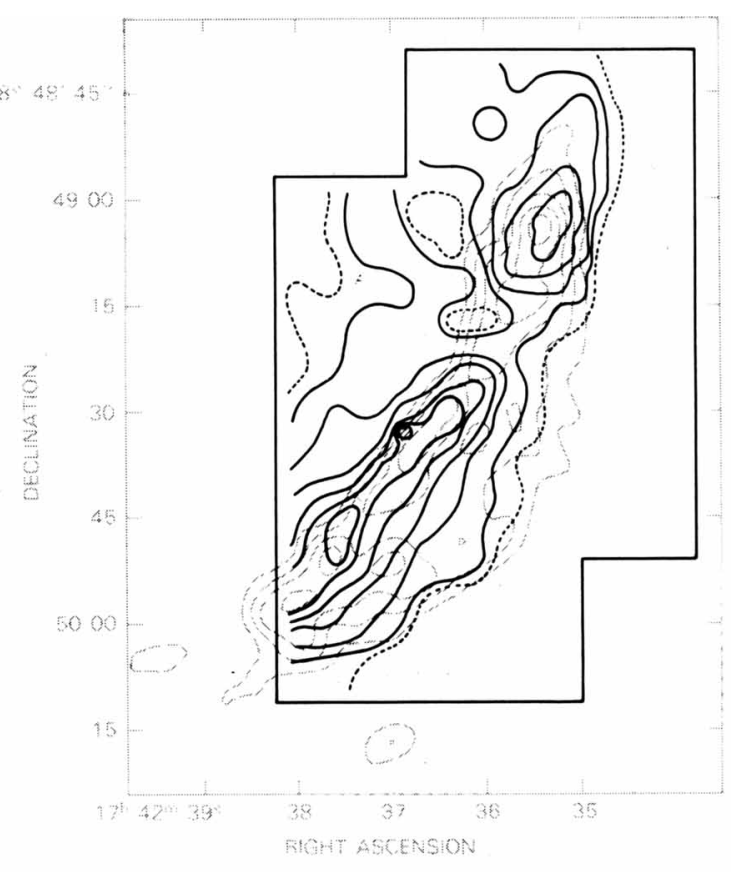

Figure 2: Contour intervals associated with the western arched filaments (G0.1+0.08) at $10 \mu \mathrm{m}$ and $6 \mathrm{~cm}$ wavelengths are set at 80 , $100, \ldots ., 240$ and 8,12 , 16, 20, $24 \mathrm{mJy} /$ beam area, respectively.

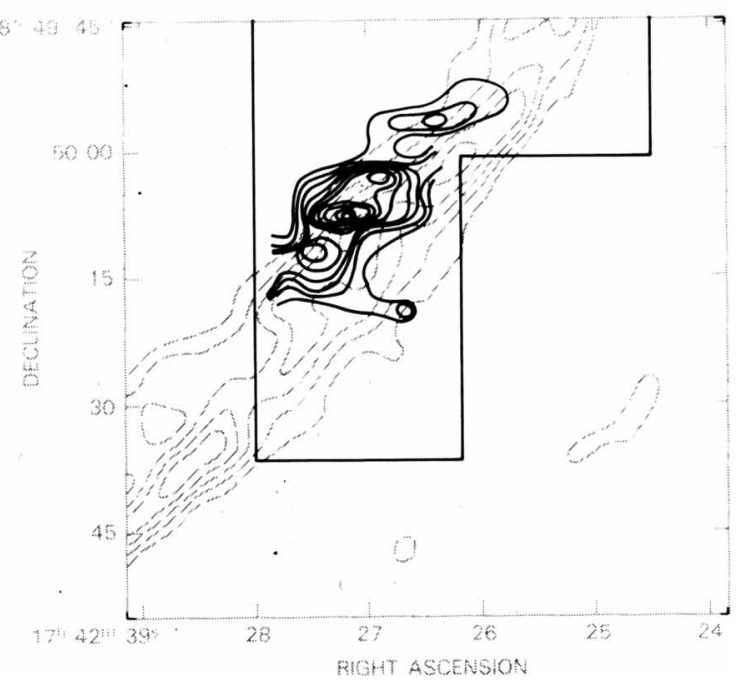


The 10 and $20 \mu \mathrm{m}$ observations were carried out at the NASA Infrared Telescope Facility at Mauna Kea. The radio maps are based on observations made using the Very Large Array (Yusef-Zadeh and Morris 1987a) at $6 \mathrm{~cm}$ wavelength and have spatial resolutions which are identical to the IR maps.

\section{The Arched Filaments (G0.1+0.08)}

The ionized features known as the eastern and the western arched filaments lie about 10' north of the Sgr A complex (see the review by Yusef-Zadeh, this conference). The northern segment of the arched filaments appears to distort the linear nonthermal filaments, which suggests that these filamentary complexes are associated with each other. Figures 1 and 2 show the intensity contours of $10 \mu \mathrm{m}$ emission from the eastern and western arched filaments superimposed on the 5$\mathrm{GHz}$ maps. The $10 \mu \mathrm{m}$ distribution is elongated along the filaments of ionized gas. Despite this general correlation between the $10 \mu \mathrm{m}$ and $6 \mathrm{~cm}$ distributions, much of the IR emission arising from the eastern arched filaments appears to be shifted eastward. The direction of the displacement of ionized gas with respect to the dust distribution is similar to the direction of the shift noted between the molecular and ionized gas distributions (Serabyn and Güsten 1987).

The 6-cm continuum radiation is known to be free-free emission from an optically thin plasma. The IR flux is much greater than expected from plasma radiation at $10 \mu \mathrm{m}$ and, therefore, is considered to be due to thermal emission from dust grains.

The ratio $\mathrm{R}$ of $10-\mu \mathrm{m}$ to $5-\mathrm{GHz}$ flux densities along the eastern arched filaments varies between 20 and 80 and is markedly higher than its western counterpart with $R \sim 10$. The spatial variation in $R$ may be due to a non-constant value of the dust-to-gas ratio along the two segments of the filaments. Alternatively, the difference may be due to differences in the nature of the heating sources which may have a softer spectrum along the eastern than the western arched filaments. If photoionization by ZAMS stars is responsible for heating the dust and gas in the filaments, the blackbody temperatures of the source of excitation along the eastern and western arched filaments are estimated to be $\sim 2.6 \times 10^{4} \mathrm{~K}$ and $\sim 3.3 \times 10^{4} \mathrm{~K}$, respectively (Panagia 1973).

\section{The Pistol (G0.15-0.05)}

The brightest radio source in the region near the prominent thermal feature G0.18-0.04, which crosses the nonthermal filaments along the Galactic equator, is known as the pistol (G0.15-0.04). Based on its unusual morphology and its kinematics, as discussed in this conference by Yusef-Zadeh, Morris and van Gorkom, this source is considered to be the site of an interaction between thermal and nonthermal filaments.

The 10- $\mu \mathrm{m}$ map of the region near G0.15-0.05 is shown in Figure 3 superimposed on the 6 - $\mathrm{cm}$ radio continuum map. We see five peaks in the $10-\mu \mathrm{m}$ map, two of which coincide with the strongest peaks seen in the $5-\mathrm{GHz}$ map. The values of $\mathrm{R}$ for these peaks, i.e., GCS5 and GCS6 (the eastern peak) in Table 1, are 25 and 50 , respectively. We also observed these two sources at $20-\mu \mathrm{m}$. Their 10 -to- $20 \mu \mathrm{m}$ color temperature is less than $112 \mathrm{~K}$. 
Figure 3: Contour intervals associated with the pistol (G0.15-0.05) at $10 \mu \mathrm{m}$ and $6 \mathrm{~cm}$ wavelengths are set at 0.4 , $0.6,0.8,1,1.2,1.4,2$, $4,8,16 \mathrm{Jy} /$ beam area and $-1,1,3,5,9,13$, $17,21,29 \mathrm{mJy} /$ beam area, respectively.
Figure 4: The thick solid lines represent the region of G-0.2-0.07 which we mapped at $10 \mu \mathrm{m}$. Contour intervals at $10 \mu \mathrm{m}$ and $6 \mathrm{~cm}$ wavelengths are set at 100, 200,400, $800,1000,1200,1400$, $1600,1800,2000$ and $5,5,10,30,50,70,90$ $\mathrm{mJy} /$ beam area, respectively.
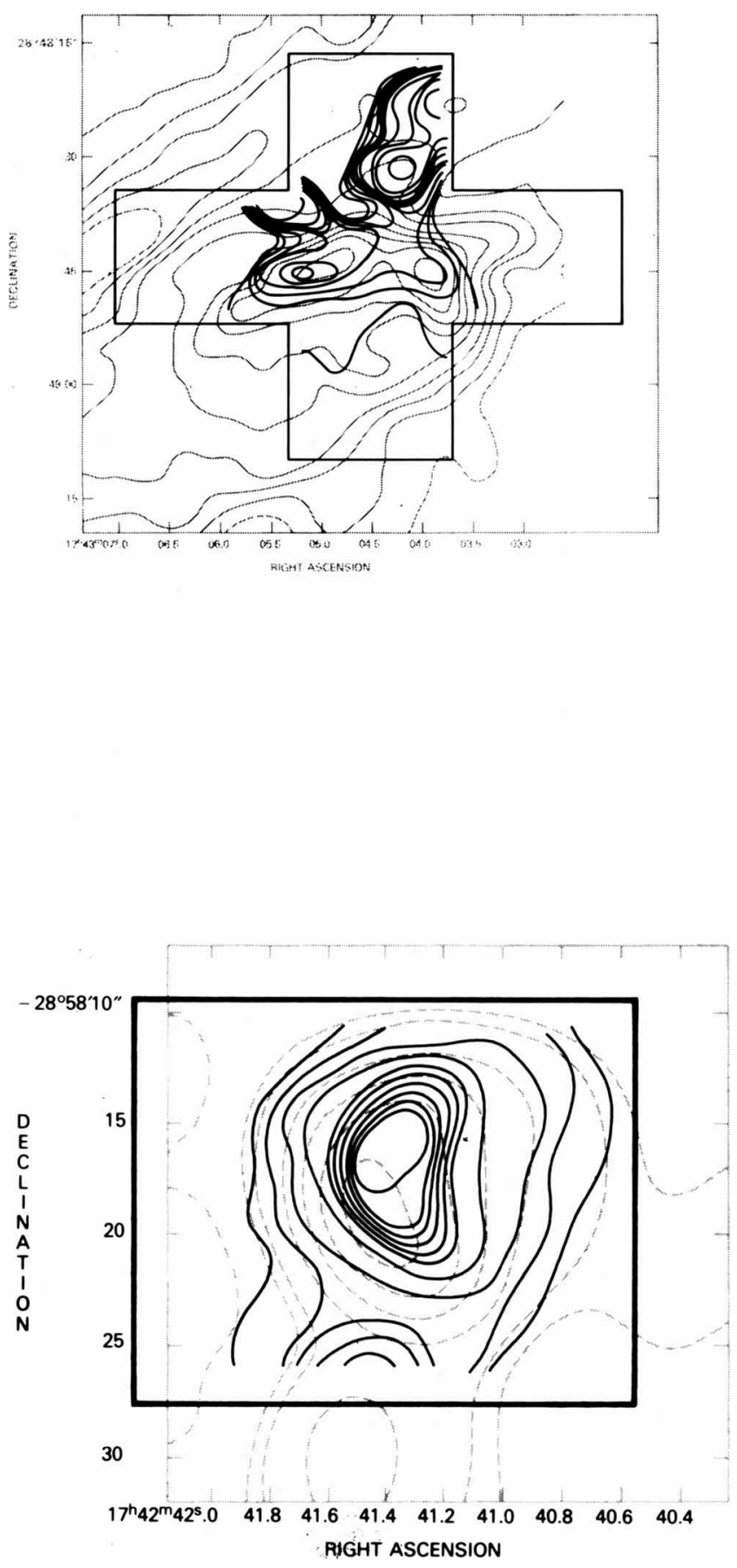
The other three IR peaks, which were first identified by Okuda et al. (this conference), are most peculiar. Their high-resolution $10-\mu \mathrm{m}$ and near-IR maps have resolved the three peaks into 5 discrete sources. These authors report that this quintuplet has a large near-IR polarization, shows deep foreground silicate absorption, and is devoid of any hydrogen recombination line emission. These characteristics have led Okuda et al. to consider the hypothesis that the quintuplet is a cluster of newly born stars near the Galactic center. If so, it is expected that this cluster is associated with the parent molecular cloud. Future high-resolution molecular observations hold a key for a further test of this hypothesis.

Alternatively, it is tempting to consider the possiblity that the quintuplet is associated with the continuum Arc and, in particular, with the pistol which shows an unusually large linewidth (total width of $\sim 90 \mathrm{~km} / \mathrm{s}$ ) and a large central velocity $(\sim 130 \mathrm{~km} / \mathrm{s}$.) The pistol is thought to be ionized by the interaction of thermal and nonthermal gas (Yusef-Zadeh and Morris 1987b). The dust, possibly small grains, may then be heated by their collision with hot relativistic particles. This process is expected to produce excess near-IR emission and a dust temperature that is considerably higher than the equilibrium value (Dwek 1986). We note that color temperatures for the three sources, as listed in Table 1, range between 500 and $1000^{\circ} \mathrm{K}$. These high values are consistent with those based on the measurements reported by Okuda et al., who account for the effects of silicate absorption at $10 \mu \mathrm{m}$ in their calculations.

\section{Sgr A East HII Region (G-0.2-0.07)}

Based on high-resolution radio continuum and radio recombination line measurements, a cluster of HII regions was found to lie at the eastern edge of the Sgr A East shell (Ekers et al. 1983; and Goss et al. 1985). Goss et al. have suggested that an $\mathrm{O} 7$ star is needed to ionize the brightest component of this HII cluster. The velocities of these HII regions suggest that they are probably associated with the $40 \mathrm{~km} / \mathrm{s}$ cloud which is known to lie near the Galactic center. Okumura-Kawabe et al. have reported in this symposium that $\mathrm{NH}_{3}$ emission from the $40 \mathrm{~km} / \mathrm{s}$ cloud is most intense in the region surrounding the HII regions.

Our contour map of the brightest component of the Sgr A East HII complex, designated as source A in Ekers et al.(1983), is shown in Figure 4. The integrated radio and IR flux densities are estimated to be 0.44 and $15.1 \mathrm{Jy}$, respectively. Therefore, the ratio $\mathrm{R} \sim 34$, which is higher than expected from typical HII regions excited by $\mathrm{O}$ stars. This suggests that the exciting star has a spectrum that is softer than that of an $\mathrm{O} 7$ star.

It is possible either that stellar ultraviolet photons are absorbed directly by dust particles in the HII region or that high-energy particles associated with $\mathrm{Sgr}$ A East contribute to the heating of the dust and in such a way as to account for the excess IR emission seen in source A. The morphologies of this HII region and Sgr A East indicate a clear connection between these two objects (Yusef-Zadeh and Morris 1987c).

\section{Conclusion}

Several of the Galactic center sources explored by us in the mid-IR show a variety of unusual characteristics, including the relative displacements of the IR 
Table 1: Color Temperatures of

Discrete IR Sources in G0.15-0.05 (P1sto1)

\begin{tabular}{lccc}
\hline Source & $\begin{array}{c}\mathrm{F}_{\nu}(10.8 \mu \mathrm{m}) \\
\mathrm{mJy}\end{array}$ & $\begin{array}{c}\mathrm{F}_{\nu}(19.2 \mu \mathrm{m}) \\
\mathrm{mJy}\end{array}$ & $\begin{array}{c}\mathrm{T}_{\operatorname{color}}(19.2 \mu \mathrm{m} / 10.8 \mu \mathrm{m}) \\
\mathrm{K}\end{array}$ \\
\hline GCS3-2 & 21,100 & & 1000 \\
GCS3-3 & 20,240 & 9,370 & 498 \\
GCS4 & 7,183 & 13,960 & $\leq 5500$ \\
GCS5 & 961 & $\geq 2390$ & $\leq 112$ \\
GCS6 & 1,447 & $\geq 3060$ & $\leq 110$ \\
\hline
\end{tabular}

and radio, a high value of the ratio of IR and radio fluxes, and very high IR color temperatures. These observations, therefore, fuel speculation that unusual processes, such as the heating of small dust grains by high-energy particles, play an important role in the Galactic center region.

We wish to thank Harvey Moseley for his initial help in this project.

\section{References}

Dwek, E. 1986, Ap.J., 302, 363.

Ekers, R., van Gorkom, J., Schwarz, J.H., and Goss, W.M. 1983, Astron.Astrophys., 122,143.

Goss, W.M., Schwarz, U.J., van Gorkom, J.H. and Ekers, R.D. 1985, M.N.R.A.S., 215, 69p.

Okuda, H., Shibai, H., Matsuhara, H., Kobayashi, Y., Kaifu, N. et al. 1988, Presented at the IAU Symposium no. 136 on the Galactic Center.

Okumura-Kawabe, S., Ishiguru, M., Fomalont, E.B., Chikada, Y., Kasuga, T., et al. 1988, Presented at the IAU Symposium no. 136 on

the Galactic Center.

Panagia, N. 1973 Astron.J., 78, 929.

Serabyn, E. and Güsten, R. 1987, Astron.Astrophys., 184, 133.

Yusef-Zadeh, F. 1988, Presented at the IAU Symposium no. 136 on the Galactic Center.

Yusef-Zadeh, F. and Morris, M. 1987a Ap.J., 322, 721.

Yusef-Zadeh, F. and Morris, M. 1987b Astron.J., 94,557.

Yusef-Zadeh, F. and Morris, M. 1987c Ap.J., 320, 545.

Yusef-Zadeh, F., Morris, M. and Chance 1984 Nature, 310, 557.

Yusef-Zadeh, F., Morris, M. and van Gorkom, J.H. 1988 Presented at the IAU Symposium no. 136 on the Galactic Center. 